Pacific Journal of Mathematics

SYMMETRIC PLANES 


\section{SYMMETRIC PLANES}

\section{RAINER LÖWEN}

Symmetric planes are defined as stable planes carrying an additional structure of a symmetric space whose symmetries are automorphisms of the plane. An example of a stable plane is the geometry induced by a topological projective plane on any of its open subsets. We consider several examples of this type which are, in fact, symmetric planes.

Working with the Lie triple system, we construct a linear local approximation to both the geometric and the differential geometric structure of a symmetric plane $M$. We show that under some reasonably mild restrictions, this so-called tangent translation plane determines the global structure of $M$ as a symmetric plane. Later, this result will be used in order to determine all symmetric planes in low dimensions. The two-dimensional case of this classification is given in the present paper. Symmetric planes often turn up inside stable planes of sufficient homogeneity, and their classification may then be applied.

Among all stable planes with a point set homeomorphic to the real affine plane, K. Strambach [24] has determined those which admit a reflection at each point. The purpose of this paper, together with two subsequent ones $[17,18]$, is to extend Strambach's result to 2-dimensional locally compact planes with an arbitrary point set and, moreover, to arbitrary 4-dimensional locally compact stable planes. (The definition of a stable plane is given below; examples are provided by all open subsets of compact projective planes.)

The present paper deals with the case where the reflections of a plane generate a transitive group of collineations containing exactly one reflection in each point. Such planes turn out to be what we call symmetric plane; that is, the reflections are the symmetries of some differentiable symmetric space structure on the point set (Theorem A). The proof depends on the fact that the collineation group of a locally compact stable plane is locally compact in the compact-open topology [15]. Motivated by this result we proceed to a systematic treatment of symmetric planes.

The main result (Theorem B) states that it is possible to construct a "linear approximation" to the local structure of a symmetric plane, called the tangent translation plane. It consists of a topological translation plane, defined on some tangent space of the symmetric space of points and compatible with the Lie triple structure present 
on that tangent space. The triple multiplication is considered as part of the structure of the tangent translation plane. Theorem C says that under some rather mild restrictions, the structure of a symmetric plane is completely determined globally by its tangent translation plane.

It would be of some interest to have a converse to Theorem B:

Problem. Does every structure composed of a topological translation plane and a Lie triple system which have the properties asserted in Theorem B arise as the tangent translation plane of some symmetric plane?

While the results $\mathrm{A}$ and $\mathrm{B}$ are rather direct consequences of known theorems on symmetric spaces, they provide the principal tool for our subsequent work. Specifically, Theorem B makes it possible to apply results on topological translation planes in the study of symmetric planes. Owing to their linear structure, translation planes are much better understood than any other class of topological affine planes. (Indeed, the most homogeneous ones have been classified in sequences of papers by $\mathrm{D}$. Betten and $\mathrm{H}$. Hähl; see $[3,9]$, and references given there. This fact will, however, not be used here.) Therefore, Theorems $\mathrm{B}$ and $\mathrm{C}$ together provide enough information to serve as a basis for our classification of 4-dimensional symmetric planes [17]. As an illustration of the techniques employed there, we give the classification of the 2-dimensional symmetric planes in the present paper. Theorem $\mathrm{A}$ is the germ of the less directly accessible result of the third paper [18], namely: any 4-dimensional locally compact stable plane which possesses reflections at each point contains a symmetric plane, and the complement is a point or a line, or empty. The proof uses the classification of symmetric planes and, this time, the classification of translation planes. Finally, all planes with a reflection at each point will be determined in [18].

In $\S 1$, we give the definition of a symmetric plane, and some easy consequences of that definition. Section 2 opens with the proof of Theorem A and continues with a list of examples of 2- and 4dimensional symmetric planes. The list contains all non-desarguesian affine translation planes and 14 open subplanes, defined by quadratic forms, of the real and complex projective planes; it is actually complete, as will be shown in Theorem $F$ of $\S 5$ for the 2-dimensional case, and in [17]. In the next section, $\S 3$, we give a brief account of all notions and results pertaining to symmetric spaces that we need in the sequel. Section 4 contains the main Theorems B and C, and a characterization of affine translation planes among all symmetric 
planes (Theorem D). Theorem $\mathrm{E}$ in the same section asserts that the global uniqueness theorem (Theorem C) applies to most of the examples given in $\S 2$.

Notation. For a group $\Sigma$ acting on a space $X$, the isotropy group of $p \in X$ will be denoted $\Sigma_{p}$. The centralizer of a subgroup $\Gamma$ in $\Sigma$ will be written $C_{\Sigma}(\Gamma)$, and the symbol $\Phi \rtimes \Psi$ will designate a semidirect product of groups, $\Phi$ being the normal factor. $\Delta^{1}$ will stand for the identity component of a topological group $\Delta$.

\section{Definitions and preliminaries.}

DeFinition 1.1. A symmetric space is a finite dimensional differentiable manifold $M$ with a differentiable multiplication $M \times$ $M \rightarrow M:(x, y) \rightarrow x \cdot y$, such that $x \cdot y$ may be interpreted as the image $x^{\sigma_{y}}$ of $x$ under an involutory diffeomorphism $\sigma_{y}$, called the symmetry around $y$, which has the properties (a) and (b):

(a) $y$ is an isolated fixed point of $\sigma_{y}$;

(b) $\sigma_{y}$ is an automorphism of the entire structure; that is, it maps $z \cdot w$ to $z^{\sigma_{y}} \cdot w^{\sigma_{y}}$.

The automorphism groups generated by all symmetries respectively, by all products $\sigma_{x} \sigma_{y}$ of two symmetries are called the groups of motions, or displacements, of the space and denoted $\Sigma$ respectively, $\Sigma^{+}$. Obviously, $\Sigma^{+}$is normal in $\Sigma$, and $\left|\Sigma / \Sigma^{+}\right| \leqq 2$.

The axiom stating that the symmetries are automorphisms may also be expressed as follows: For $x \in M$ and $\alpha \in \Sigma$ one has $\sigma_{x}^{\alpha}=\sigma_{x^{\alpha}}$, where $\sigma_{x}^{\alpha}$ denotes the conjugate $\alpha^{-1} \sigma_{x} \alpha$.

Concerning symmetric spaces, we use the terminology of [14]. Results which we use without proof can mostly be found there.

Definition 1.2. A stable plane $(M, \mathscr{L})$ consists of a Hausdorff space $M$ together with a system $\mathscr{L}$ of subsets of $M$, called lines, and a topology on $\mathscr{L}$ such that the following properties (a), (b), and (c) hold:

(a) any two distinct points (=elements of $M$ ) $x, y$ are on a unique line $x \cup y$ depending continuously on $(x, y)$;

(b) the pairs of distinct lines that do intersect form an open subset $\mathscr{D}$ of $\mathscr{L} \times \mathscr{L}$ ("axiom of stability");

(c) $\mathscr{D}$ is mapped continuously into $M$ under intersection.

The pencil of all lines containing $x \in M$ is denoted $\mathscr{L}_{x}$. A continuous collineation (also termed automorphism or simply collineation) of a stable plane is a homeomorphism of the point set that takes lines into lines. Collineations form a group $\Gamma$, which is usually given the compact-open topology. An involutory collineation $\gamma$ is 
called a reflection at a point $x \in M$ if it fixes each line through $x$. The point $x$ is then called the centre of $\gamma$.

By the dimension of a stable plane we shall always mean the topological dimension of its point set. Locally compact stable planes of positive dimension have been studied in $[15,16]$. The reader is referred there for more information.

Definition 1.3. Let $M$ be a Hausdorff space and assume that there is given a structure $(x, y) \rightarrow x \cdot y$ of a symmetric space of (finite) positive dimension and a structure $(M, \mathscr{L})$ of a stable plane on $M$. If the two structures are compatible, in the sense that all symmetries $\sigma_{x}$ are collineations of the plane $(M, \mathscr{L})$, then $M$ together with these structures is called a symmetric plane.

Proposition 1.4. The symmetry at a point $x$ of a symmetric plane is a reflection in the geometric sense (see 1.2).

This will follow from the following more general fact.

LEMMA 1.5. Let $\sigma$ be an involutory collineation of a locally compact positive dimensional stable plane $(M, \mathscr{L})$. If $\sigma$ has an isolated fixed point $x$, then $\sigma$ is a reflection at $x$.

Proof. If $p \in M$ is not fixed under $\sigma$, then $L_{p}:=p \cup p^{\sigma}$ is a fixed line, which depends continuously on the nonfixed point $p$. As $p$ approaches $x$, the lines $L_{p}$ accumulate at some fixed line $L$ through $x$, by $[15: 1.5,1.17]$.

(2) By [15:1.7, 1.12], the space $\mathscr{L}_{x} \mid\{L\}$ is acyclic. A theorem of P. A. Smith [23] therefore gives us a second fixed line $K$ through $x$.

(3) Assume that $\sigma$ operates nontrivially on $\mathscr{L}_{x}$. We may then take for $K$ a fixed line which is a limit of nonfixed lines $K_{x} \in \mathscr{L}_{x}$. $K$ contains a nonfixed point $p$. Choose a sequence $p_{n} \in K_{n}$ converging to $p$. Then for large $n$ the intersections $L_{p_{n}} \cap L \neq x$ exist and converge to $x$, contrary to the assumption that $x$ be isolated.

Proposition 1.6. Let $p$ be a point of a symmetric plane $M$. Then the isotropy group $\Sigma_{p}$ of $p$ in the motion group $\Sigma$ is equal to the centralizer of $\sigma_{p}$.

Proof. In any symmetric space one has $\Sigma_{p} \leqq C_{\Sigma}\left(\sigma_{p}\right)$, because motions are automorphisms. For the converse inclusion, one notes that each image of $p$ under a collineation centralizing $\sigma_{p}$ is a centre 
of $\sigma_{p}$, and that a nontrivial collineation cannot have more than one centre.

1.7. Remark on the topology of the motion group. The collineation group $\Gamma$ of a connected symmetric plane $M$ is point transitive, since the same is true of the motion group $\Sigma \leqq \Gamma$, see [14: p. 91]. $\Gamma^{1}$ is a second countable locally compact transformation group of $M$ with respect to the compact-open topology $T$ [15: $\S 2]$, hence is a Lie group $[20,26]$. On the other hand, $\Sigma^{+}$is also a Lie transformation group of $M$ in some topology $T_{1}$ [14: p. 88]. By [1], $T_{1}$ is finer than the topology induced on $\Sigma^{+}$by $T$, and the inclusion $\Sigma^{+} \rightarrow \Gamma^{1}$ is continuous with respect to the topologies $T_{1}, T$. Therefore, $\Sigma^{+}$is a Lie subgroup of $\Gamma^{1}$ by [10: p. 84], and is in fact the smallest Lie subgroup of $\Gamma^{1}$ containing all products of two symmetries. If $\Sigma^{+}$ happens to be closed in $\Gamma$, then the topologies $T$ and $T_{1}$ coincide on $\Sigma^{+}$.

We shall always think of $\Sigma^{+}$as carrying the topology $T_{1}$. Occasionally, we shall apply to $\Sigma^{+}$theorems on collineation groups which have been formulated in $[15,16]$ referring to the compactopen topology. This is justified, since most of those theorems hold equally well for any locally compact group acting continuously as a group of collineations.

2. Examples. The only effective way of introducing a symmetric structure on a stable plane is to derive it from a transitive group action. The procedure is described in the following theorem. Let $(M, \mathscr{L})$ be a locally compact stable plane of positive dimension, and let $\Delta \leqq \Gamma$ be a closed subgroup of the collineation group, with $\Delta / \Delta^{1}$ compact. Alternatively, let $\Delta \leqq \Gamma$ be a Lie group acting topologically on $M$. Then we have

2.1. THEOREM A. In addition to the above hypotheses, assume that the group $\Delta$ is transitive on the stable plane $M$ and that some isotropy group $\Delta_{0}$ contains in its centre a reflection $\rho_{o}$ at the point $o$.

Then $(M, \mathscr{L})$ may be given the structure of a symmetric plane in such a way that the symmetry at a point $y$ is equal to the unique reflection $\rho_{y} \in \Delta$ at $y$ conjugate to $\rho_{o}$.

REMARK. (a) Note that the motion group of $M$ may be a proper (normal) subgroup of $\Delta$.

(b) If $\Delta$ contains only one reflection at $o$ then the hypothesis $\rho_{o} \in C\left(\Delta_{o}\right)$ is evidently satisfied. This situation will occur frequently. 
Proof. The full collineation group $\Gamma$ is locally compact and has a countable basis for its topology [15: §2]. Furthermore $M$ is locally contractible [15: 1.12]. Therefore, any transitive closed subgroup $\Delta \leqq \Gamma$, with $\Delta / \Delta^{1}$ compact, is a Lie group by [26]. It is only the latter property of $\Delta$ which we use in the sequel.

Our hypothesis and the uniqueness of the centre for any nontrivial central collineation now imply that $\Delta_{o}=C_{\Delta}\left(\rho_{o}\right)$. By [14: p. $73]$, the coset space $\Delta / \Delta_{0}$ becomes a symmetric space with the quotient differentiable structure if one defines the symmetric structure by

$$
\Delta_{o} \delta \cdot \Delta_{o} \gamma:=\Delta_{o} \rho_{o} \delta \gamma^{-1} \rho_{o} \gamma \text {. }
$$

The homeomorphism

$$
\Delta / \Delta_{o} \longrightarrow M: \Delta_{o} \delta \longrightarrow o^{\delta}
$$

takes this product into the multiplication defined by $x \cdot y:=x^{\rho y}$.

Affine translation planes will be the first example of symmetric planes illustrating the situation described in Theorem A. Other, less trivial examples will follow. There is no hope, however, of obtaining any new information on translation planes by introducing this additional structure. In fact, in translation planes, there is no interplay at all between the geometric and symmetric structures. As symmetric spaces, all translation planes of a given dimension are isomorphic. Yet there are a vast number of nonisomorphic translation planes, see $[3,9,13]$.

Instead of a definition of translation planes we give here the following description, which covers all locally compact connected affine translation planes (cf. [22: 7.23; 11: VII. 3]): They may be obtained as stable planes defined on the point set of real affine space $E^{2^{n}}$ of dimension $2^{n}$, where $1 \leqq n \leqq 4$, with a translation invariant system of $2^{n-1}$-dimensional affine subspaces as the set $\mathscr{L}$ of lines. Of course, $\mathscr{L}$ may be recovered from the pencil $\mathscr{L}_{0}$ of lines through a point $o$, the origin, say. The condition for any set $\mathscr{L}_{0}$ of $2^{n-1}$ dimensional subspaces through $o$ to be the pencil for a (unique) topological translation plane is that (a) any point other than $o$ be on a unique element of $\mathscr{L}_{0}$ and that (b) $\mathscr{L}_{0}$ be compact in the topology induced by the Grassmann manifold of $2^{n-1}$-dimensional vector subspaces of $\boldsymbol{R}^{2^{n}}$; cf. [5].

Proposition 2.2. Each locally compact connected affine translation plane is in a natural way a symmetric plane, and the motion group coincides with the group $\boldsymbol{R}^{2^{n}}$ of all translations of $E^{2^{n}}$.

Proof. We identify $E^{2^{n}}$ with the real vector space $\boldsymbol{R}^{2^{n}}$ by 
choosing an origin $o$. The system $\mathscr{L}_{o}$ is always invariant under multiplication by real scalars, and so is the union of its translates $\mathscr{L}$. The map $\sigma_{0}:=(x \rightarrow-x)$ is a reflection, and the group

$$
\Delta=\left\{x \longrightarrow \pm x+y ; y \in \boldsymbol{R}^{2^{n}}\right\}
$$

has all the properties used in Theorem A. Since $\sigma_{0} \sigma_{y}=(x \rightarrow x+2 y)$, the motion group $\Sigma^{+}$consists of all translations.

REMARK. The dilation groups of an affine translation plane are isomorphic to the multiplicative group of its kernel, which is one of the three classical (skew) fields; cf. [11: 7.8]. Therefore, the reflection at any point is uniquely determined, and so is the structure of symmetric plane generated by the reflections.

Definition 2.3. Let $K$ be one of the three classical (skew) fields $\boldsymbol{R}, \boldsymbol{C}$, and $\boldsymbol{H}$, and let $\alpha: \boldsymbol{K} \rightarrow \boldsymbol{K}$ be the identity or conjugation (in $\boldsymbol{K}=\boldsymbol{C}$ or $\boldsymbol{H}$ ). Further let $f$ be any hermitian sesquilinear form on $\boldsymbol{K}^{3}$ with respect to $\alpha$. We allow $f$ to be degenerate. If $f$ is positive definite on some one-dimensional subspace of $K^{3}$, we define $M_{f}$ to be the open subplane of $P_{2} K$ whose points are all one-dimensional subspaces with this property. In the case $\boldsymbol{K}=\boldsymbol{C}, \alpha=\mathrm{id}$, where this definition does not apply, let $M_{f}$ be the set of nonisotropic onedimensional subspaces. The lines of an open subplane $N \cong P_{2} K$ are, by definition, the intersections $N \cap L$ with the lines $L$ of $P_{2} K$. The plane $M_{f}$ will be called the plane defined by the hermitian form $f$.

Proposition 2.4. The plane $M_{f}$ defined by a hermitian form $f$ over $\boldsymbol{K}=\boldsymbol{R}, \boldsymbol{C}$ or $\boldsymbol{H}$ is a connected symmetric plane.

In fact, the projective unitary group $\mathrm{PU}(f)$ satisfies all requirements of Theorem A. The motion group $\Sigma^{+}$is a connected normal Lie subgroup of PSU $(f)$. In particular, if $f$ is nondegenerate, $\Sigma^{+}$ is the the simple connected component $\mathrm{PSU}(f)^{1}$.

Proof. Transitivity of the unitary group follows from a theorem of Witt. To apply Theorem A, one uses existence and uniqueness of unitary reflections at each point of $M_{f}$. The details are left to the reader.

We wish to describe in some detail the various possible examples of this type for the complex and real cases. We shall need this material later in our proof that the given list of examples is complete [17]. We start with planes defined by complex nondegenerate forms, where the motion group is known from the preceding proposition. 
For $a, b, c \in C$, we define on $C^{3}$ a hermitian form, and a symmetric one, as follows.

$$
\begin{aligned}
& f_{a, b, c}\left(z_{1}, z_{2}, z_{3}\right):=a z_{1} \bar{z}_{1}+b z_{2} \bar{z}_{2}+c z_{3} \bar{z}_{3} \\
& g_{a, b, c}\left(z_{1}, z_{2}, z_{3}\right):=a z_{1}^{2}+b z_{2}^{2}+c z_{3}^{2}
\end{aligned}
$$

2.5. If $f=f_{1,1,1}$, then $M_{f}$ consists of the entire point set of $P_{2} C$ and is called the complex elliptic plane. The motion group $\Sigma^{+}=$ $\mathrm{PSU}_{3}(\boldsymbol{C}, 0)=\Sigma$ is compact and 8-dimensional.

2.6. If $f=f_{-1,1,1}$, then $M_{-f}$ is the open unit ball in the affine plane $z_{1}=1$ (the complex hyperbolic plane, cf. [15: §7; 4]), and $M_{f}$ is the exterior of $M_{-f}$ in $P_{2} C$. Their common motion group $\Sigma^{+}=$ $\mathrm{PSU}_{3}(\boldsymbol{C}, 1)=\Sigma$ is 8-dimensional and noncompact. Sometimes we employ the term "hyperbolic plane" for $M_{f}$ as well, and use the specifications interior and exterior to distinguish between $M_{-f}$ and $M_{f}$.

2.7. If $g=g_{1,1,1}$, then $\Sigma^{+}=\mathrm{PSO}_{3} C=\Sigma$ is isomorphic to $\mathrm{PSL}_{2} C$. To describe the geometric features of $M_{g}$, we remark that the complement $P_{2} C \backslash M_{g}$ is a topological oval, cf. [6]. $M_{g}$ will be called the complex oval plane.

We continue with the degenerate forms, where it takes a few computations to determine the motion groups. The following preparatory definition will be useful:

Definition 2.8. Assume that a hermitian form $f$ on $\boldsymbol{K}^{\mathbf{3}}$ is expressed as $f\left(z_{1}, z_{2}, z_{3}\right)=f^{\prime}\left(z_{1}, z_{2}\right)$, where $f^{\prime}$ is a nondegenerate form in two variables. Then define

$$
\Phi_{f}^{+}=\left\{\left(\begin{array}{cc}
A & U^{t} \\
0 & 1
\end{array}\right) ; A \in \mathrm{SU}\left(f^{\prime}\right), \quad U \in \boldsymbol{K}^{2}\right\} .
$$

Clearly, $\Phi_{f}^{+} \leqq \mathrm{SU}(f)$. Further, let the subgroups $\Psi_{f} \cong \mathrm{SU}\left(f^{\prime}\right)$ and $T \cong K^{2}$ of $\Phi_{f}^{+}$be defined by $U=0$ and $A=1$, respectively. Note that $T$ is normal.

2.9. Let $f=f_{1,1,0}$. Then there is exactly one isotropic onedimensional subspace $\infty=\langle 0,0,1\rangle$. The complement is the punctured complex projective plane $M_{f}=P_{2} C \backslash\{\infty\}$. We claim that $\Sigma^{+}=P \Phi_{f}^{+}$ and $\Sigma=P \Phi_{f}$, where $\Phi_{f}=\Phi_{f}^{+} \rtimes\langle\alpha\rangle$, and

$$
\alpha=\left(\begin{array}{lll}
1 & & \\
& 1 & \\
& & i
\end{array}\right) \text {. }
$$


$T$ is the radical of $\Phi_{j}^{+}$, and $\Psi_{f} \cong \mathrm{Spin}_{3}$, a Levi complement, acts irreducibly on $T$.

Proof. Note first that the action of $\Psi_{f}$ on $T$ is contragredient to the standard representation of $\mathrm{SU}_{2}$, hence is irreducible. $P \Phi_{f}$ is easily seen to be transitive on $M_{f}$. Indeed, $T$ consists of all elations with centre $\infty$, and the action of $\Psi_{f}$ on the $x$-axis $\left(z_{3}=0\right)$ is the transitive action of $\mathrm{PSU}_{2} C$ on the projective line $P_{1} C$. Therefore, we have $\Sigma=P \Delta \leqq P \Phi_{f}$, where $\Delta \leqq \Phi_{f}$ is the Lie subgroup generated by the reflection

$$
\sigma=\left(\begin{array}{lll} 
& i & \\
i & & \\
& & i
\end{array}\right) \in \Phi_{f}
$$

at the point $\langle 1,-1,0\rangle$ together with its conjugates in $\Phi_{f} ;$ cf. 2.4 and 1.7.

In fact, we can see that $\Delta=\Phi_{f}$, since the conjugates of $\sigma$ under $\Psi_{f}$ generate the group $\Omega:=\Psi_{f} \times\langle\alpha\rangle$, by simplicity of $\Psi_{f}$. Indeed, normality of $\Delta$ and irreducibility of the action of $\Psi_{f}<\Omega<\Delta$ on $T$ together imply that the Lie algebra of $\Delta$ contains that of $T$.

2.10. Let $f=f_{1,-1,0}$. Then the two stable planes

$$
M_{f}=\left\{\left(1, z_{2}, z_{3}\right) ;\left|z_{2}\right|<1\right\}
$$

and $M_{-f}$ are isomorphic. $M_{f}$ is called the complex cylinder plane; this geometry has been discussed in [15: 7.3]. The motion groups are given by $\Sigma=P \Phi_{f}$ and $\Sigma^{+}=P \Phi_{f}^{+}$, where $\Phi_{f}=\Phi_{f}^{+} \rtimes\langle\sigma\rangle$, and

$$
\sigma=\left(\begin{array}{lll}
i & & \\
& -i & \\
& & -i
\end{array}\right) .
$$

Here again, $T$ is the radical, and $\Psi_{f} \simeq \mathrm{SL}_{2} \boldsymbol{R}$, a Levi complement, acts on $T$ with two irreducible invariant subspaces $T_{1}, T_{2}$. These consist of the elements given by $U=(u, \bar{u})$ and $U=(u,-\bar{u})$, respectively.

The proof is similar to the preceding one. Note that $\sigma$ is the reflection at the origin $\langle 1,0,0\rangle$, and that $\Psi_{f}$ is transitive on the $x$-axis $\left\{\left\langle 1, z_{2}, 0\right\rangle ;\left|z_{2}\right|<1\right\}$ of $M_{f}$; in fact, there, $\Psi_{f}$ induces the real hyperbolic motion group $\mathrm{PO}_{3}^{+}(\boldsymbol{R}, 1)$.

2.11. Let $g=g_{0,1,1}$. For technical reasons, we prefer instead 
of $g$ to use the equivalent form $h\left(z_{1}, z_{2}, z_{3}\right)=z_{1} z_{2}$. Then $M_{h}=$ $P_{2} C \backslash L_{\infty} \backslash Y$, where $L_{\infty}$ is the line at infinity $\left(z_{1}=0\right)$, and $Y$ is the $y$-axis $\left(z_{2}=0\right) . \quad M_{h}$ is called the complex Minkowski plane. The motion groups are $\Sigma^{+}=P \Phi_{h}^{+}$and $\Sigma=P \Phi_{h}$, where $\Phi_{h}=\Phi_{h}^{+} \rtimes\langle\sigma\rangle$, and

$$
\sigma=\left(\begin{array}{lll}
0 & 1 & \\
1 & 0 & \\
& & -1
\end{array}\right)
$$

The subgroup $\Psi_{h}$ induces on the commutator subgroup $T$ of $\Sigma^{+}$the group

$$
\left\{\left(\begin{array}{cc}
c^{-1} & \\
& c
\end{array}\right) ; c \in C^{\times}\right\}=\mathrm{SU}\left(h^{\prime}\right)
$$

of complex linear transformations.

Sketch of proof. $P \Phi_{h}$ is transitive on $M_{f}$ and contains the reflection $\sigma$ at the point $\langle 1,1,0\rangle$, which induces inversion on $\Psi_{h}$. Using the latter fact, it is easy to see that the group generated by the conjugates of $\sigma$ contains $\Psi_{h}$ and hence $\Phi_{h}$.

2.12. If $f=f_{1,0,0}$ and $g=g_{1,0,0}$, then $M_{f}=M_{g}$ is the affine plane $z_{1} \neq 0$. We call $M_{f}$ the complex euclidean plane.

2.13. We conclude with a brief discussion of the case $\boldsymbol{K}=\boldsymbol{R}$, the real field. Again let $g_{a, b, c}$ be the symmetric form given by $g_{a, b, c}\left(x_{1}, x_{2}, x_{3}\right)=a x_{1}^{2}+b x_{2}^{2}+c x_{3}^{2}$. For $g=g_{1,1,1}$, we get the elliptic plane $M_{g}=P_{2} R$, with motion group $\mathrm{PSO}_{3}$. The form $g=g_{-1,1,1}$ yields the real hyperbolic plane $M_{-g}$, and its exterior $M_{g}$, both with motion group $\Sigma^{+}=\mathrm{PO}_{3}(\boldsymbol{R}, 1)^{1}$ (but with $\Sigma=\mathrm{PO}_{3} \neq \Sigma^{+}$in the latter case). For $g=g_{1,1,0}$ we obtain the real punctured projective plane $M_{g}=$ $P_{2} R \backslash\{\langle 0,0,1\rangle\}$. The motion group $\Sigma$ is dual to the group of isometries of the euclidean affine plane. The plane $M_{g}$ for $g=g_{1,-1,0}$ is the strip between two parallel affine lines; we call it the real cylinder plane. Its motion group is isomorphic to the extension of $\boldsymbol{R}^{2}$ by the one-parameter group

$$
\left\{\left(\begin{array}{cc}
t & \\
& t^{-1}
\end{array}\right) ; 0<t \in \boldsymbol{R}\right\}
$$

of automorphisms. Finally, $M_{g}$ for $g=g_{1,0,0}$ is the real euclidean plane.

2.14. It should perhaps be added that among the Examples 2.5 
through 2.13, only six are Riemannian symmetric spaces, namely, the real and complex euclidean, elliptic, and interior hyperbolic planes. Clearly, non-desarguesian translation planes are Riemannian as well. (Note that a symmetric space is Riemannian if and only if the isotropy group $\Sigma_{0}$ is compact [14: p. 148].)

3. Summary of facts concerning symmetric spaces. Let $M$ be a connected symmetric space. In all symmetric spaces a base point, denoted $o$, is assumed to be preassigned. Morphisms are to preserve base points.

3.1. The motion group $\Sigma$ is transitive on $M$ [14: p. 91]. An easy proof may be based on the inverse function theorem, observing that $p \rightarrow o \cdot p$ has a nonsingular tangent map at $o[14: p .76]$. Moreover, $\Sigma$ is a Lie transformation group of $M$ [14: p. 88].

3.2. The isotropy group $\Sigma_{o}$ is contained in the centralizer $C\left(\sigma_{o}\right)$. Equality holds in symmetric planes (1.6). In general, the orbit of $o$ under $C\left(\sigma_{0}\right)$ consists of isolated fixed points of $\sigma_{0}$. Therefore, $\Sigma_{0}$ and $C\left(\sigma_{o}\right)$ have the same Lie algebra.

3.3. The symmetry $\sigma_{o}$ induces on the Lie algebra $\subseteq$ of $\Sigma$ the automorphism Ad $\sigma_{o}=T_{1} \theta$ of order two, where $\theta$ denotes the inner automorphism of $\Sigma$ induced by $\sigma_{o}$, and $T_{1} \theta$ denotes the tangent map at $1 \in \Sigma$. Let $\widetilde{S}^{+}$and $\widetilde{S}^{-}$be the eigenspaces corresponding to the eigenvalues 1 and -1 of $\operatorname{Ad} \sigma_{\circ}$. The following relations are easily observed.

$$
\mathfrak{S}^{+} \leqq \subseteq,\left[\mathfrak{S}^{+}, \mathfrak{S}^{-}\right] \subseteq \mathfrak{S}^{-},\left[\mathfrak{S}^{-}, \mathfrak{S}^{-}\right] \subseteq \mathfrak{S}^{+}
$$

3.4. From 3.2, we infer that $\widetilde{S}^{+}$is the Lie algebra of $\Sigma_{o}$, using that $\theta=\log \circ T_{1} \theta \circ \exp$ on an exponential neighborhood in $\Sigma$. Consequently, $\operatorname{dim} \mathfrak{S}^{-}=\operatorname{dim} \Sigma / \Sigma_{0}=\operatorname{dim} M$.

3.5. (a) By [14: p. 91], 3.3 may be sharpened as follows:

$$
\left[\mathfrak{S}^{-}, \mathfrak{S}^{-}\right]=\mathfrak{S}^{+} \text {. }
$$

This expresses in the Lie algebra the fact that $\Sigma$ is generated by the conjugacy class of $\sigma_{0}$. Actually, a proof may easily be given by observing that $\mathfrak{S}^{-} \oplus\left[\mathfrak{S}^{-}, \mathfrak{S}^{-}\right]$is an Ad $\sigma_{o}$-invariant ideal in $\mathfrak{S}^{\text {. This }}$ implies

$$
\operatorname{dim} \subseteq \leqq \frac{1}{2} n(n+1)
$$




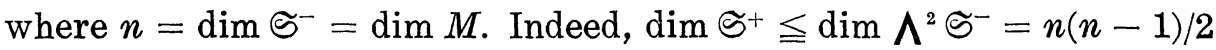
by (a); here, $\Lambda^{2}$ denotes the exterior power.

(c) Again by (a), $\mathfrak{S}$ is abelian if and only if $\mathfrak{S}=\mathfrak{S}^{-}$

3.6. $\mathfrak{S}^{+}$contains no ideal of $\subseteq$, since $\Sigma$ operates effectively on $M$.

DEFINITION 3.7. We call Lie algebra with involution any pair $(\Re, \alpha)$ consisting of a Lie algebra $\Re$ and an automorphism $\alpha$ of $\Re$, of order two. $(\Re, \alpha)$ will be called a reflection algebra if $\Re$ is generated by $\Re^{-}$(property $3.5 \mathrm{a}$ ), and an effective reflection algebra if in addition $\Re^{+}$contains no ideal of $\Re$ (property 3.6). We say that a reflection algebra $(\Re, \alpha)$ is of type $(p, n)$, if $\operatorname{dim} \Re^{+}=p$, $\operatorname{dim} \mathfrak{R}^{-}=n$.

3.8. If $(\Re, \alpha)$ is a Lie algebra with involution, then $\Re^{-}$becomes a Lie triple system if endowed with the trilinear multiplication $[x, y, z]:=[[x, y], z]$. (For the axioms, see [14: p. 78].) An effective reflection algebra $(\Re, \alpha)$ is uniquely determined by its Lie triple system. More precisely, if $\mathfrak{A}$ is the vector space spanned by the set of endomorphisms $z \rightarrow[x, y, z]$ of $\Re^{-}$, where $x, y \in \Re^{-}$, then $\Re$ is isomorphic to the algebra $\mathfrak{A} \oplus \mathfrak{R}^{-}$with the obvious multiplication, under an isomorphism fixing $\mathfrak{R}^{-}$and sending $\mathfrak{R}^{+}$onto $\mathfrak{A}$; this Lie algebra is called the standard embedding of $\Re^{-}$. The easy proof uses the fact that the centralizer of $\Re^{-}$in $\Re^{+}$is an ideal in $\Re$.

3.9. The triple system $\mathcal{S}^{-}$of $M$ may be identified as a vector space with the tangent space $T_{0} M$, in such a way that the tangent map $T_{\circ} \varphi$ of each morphism $\varphi$ of symmetric spaces becomes a morphism of triple systems. We shall always make this identification and choose the notation $\mathcal{S}^{-}$or $T_{0} M$ according to the aspect prevailing in a particular situation.

3.10. There is an exponential function $\operatorname{Exp}: T_{0} M \rightarrow M$, which is a local diffeomorphism around 0 , and which commutes with morphisms [14: Chapters I. 2 and II. 2]; that is,

$$
\operatorname{Exp} \circ \varphi=T_{\diamond} \rho \circ \operatorname{Exp} \text {. }
$$

With respect to the above identification of $T_{0} M$ and $\mathfrak{S}^{-}$, the exponential function is related to the exponential function exp: $\subseteq \rightarrow \Sigma$ of $\Sigma$ by the formula

$$
\operatorname{Exp} x=o^{\operatorname{cxp} x}
$$

which holds for all $x \in T_{0} M=\varsigma^{-}$[14: p. 95]. 
With some loss of generality, but still general enough for most of our purposes, the facts 3.9 and 3.10 can be easily deduced from similar results about Lie groups: Consider the projection $p=p_{M}$ : $\Sigma \rightarrow M: \sigma \rightarrow o^{\sigma}$. Identify $\mathcal{S}^{-}$with $T_{0} M$ via the restriction of the tangent map $T_{1} p: \subseteq=T_{1} \Sigma \rightarrow T_{0} M$. A surjective morphism $\varphi: M \rightarrow N$ induces a morphism $\bar{\varphi}: \Sigma_{M} \rightarrow \Sigma_{N}$ of motion groups in an obvious way, and $p_{M} \circ \varphi=\bar{\varphi} \circ p_{N}$. By differentiating this equation one gets 3.9 , since $T_{1} \bar{\phi}$ is a morphism of reflection algebras. Using the identification $\mathcal{S}^{-}=T_{0} M$, one defines the exponential map by the relation $3.10 \mathrm{~b}$, and one infers $3.10 \mathrm{a}$ for surjective morphisms from the fact that exp commutes with group morphisms.

3.11. The centre congruence $C(M)$ may be described [14: p. 134] as the equivalence relation

$$
\left\{(x, y) ; \sigma_{x} \sigma_{y} \in C\left(\Sigma^{+}\right)\right\}
$$

on $M$. The centre $C_{o}(M)$ of $M$ is the equivalence class of the base point.

3.12. In connection with the centres, the Lie triple systems play the same role for symmetric spaces as Lie algebras do for Lie groups. Namely, assigning to $M$ the triple system $\mathfrak{S}^{-}$sets up a one-to-one correspondence between all simply connected symmetric spaces and all Lie triple systems [14: p. 116]. Moreover, among the symmetric spaces with a given Lie triple system $\mathfrak{S}^{-}$, there is a unique simply connected one, $\widetilde{M}$, and (provided the centre of $\subseteq$ is zero) a unique centre-free one, the quotient $\widetilde{M} / C(\widetilde{M})$. Furthermore, for any other symmetric space $N$ with the same triple system, there are covering morphisms $\widetilde{M} \rightarrow N \rightarrow \widetilde{M} / C(\widetilde{M})$. In addition, these may be so chosen as to induce any preassigned isomorphism of the triple systems. A proof of these facts may be pieced together from [14: pp. 115, 135, 136, 177].

3.13. Each closed subset $N \cong M$ with the property that $N \cdot N \subseteq N$ is a symmetric subspace of $M$; that is, $N$ is a differentiable submanifold which is a symmetric space with respect to the induced multiplication [14: p. 125].

3.14. By 3.9 , the tangent space $T_{o} N$ of a subspace $N \cong M$ containing the base point is a sub-Lie triple system of $T_{0} M$. Conversely, given a subsystem $\Re \leqq T_{\diamond} M$, there is a unique connected subspace $N \leqq M$ with $\Re=T_{0} N$. It may be obtained as the orbit of $o$ under the group $\Delta \leqq \Sigma^{+}$whose Lie algebra is the subalgebra $\langle\Re\rangle \leqq \subseteq$ generated by $\Re[14:$ pp. 121, 122]. 
4. The tangent translation plane of a symmetric plane. We begin with a lemma on stable planes that will be essential in the proof of Theorems B and C:

LEMma 4.1. Let $(M, \mathscr{L})$ be a locally compact stable plane of positive dimension, and $U$ an open neighborhood of $p \in M$. Then the union $V$ of the connected components of $p$ in $L \cap U$, for all $L \in \mathscr{L}_{p}$, is also an open neighborhood of $p$.

Proof. If $V$ were not a neighborhood there would be a convergent sequence $p_{n} \rightarrow p$, such that $p_{n}$ and $p$ are in different components of $U \cap\left(p \cup p_{n}\right)$. By compactness of $\mathscr{L}_{p}$ [15: 1.17], we may assume that the lines $L_{n}=p \cup p_{n}$ converge to a line $L \in \mathscr{L}_{p}$. Choose a point $q$ not on $L$, nor on any $L_{n}$, and project $\left\{p_{n}\right\}$ into $L$ from $q$. The image sequence $\left\{p_{n}^{\prime}\right\}$ would converge to $p$. Now $L$ is locally connected [15: 1.11]. Hence by stability, $p$ has a connected neighborhood $W$ in $L$ such that $W$ can be projected from $q$ back into each $L_{n}$ within $U$. Almost all the points $p_{n}^{\prime}$ would lie in $W$, a contradiction.

Openness of $V$ is proved in a similar way. Indeed, given $v \in V$, there is a path $P \subseteq U \cap(p \cup v)$ joining $p$ to $v$. Using a compactness argument, one sees that from a point $q \notin L=p \cup v$, both $p$ and some small neighborhood $T$ of $v$ in $L$ may be projected inside $U$ into each line $K \in \mathscr{L}_{p}$ sufficiently close to $L$. The images of $P$ and $T$ are contained in $V$ and cover a neighborhood of $v$ in $M$.

Proposition 4.2. Each line of a symmetric plane is a symmetric subspace of the point set.

Proof. A line $L$ is easily seen to be closed. Since $L$ is invariant under the symmetry at each of its points (1.4), the assertion follows from 3.13 .

This fact allows to construct the tangent translation plane. Before we do this, let us note the following consequence of 4.2 , which could also be viewed as a corollary to Theorem B and known results on topological translation planes [22: 7.22].

CoROLlary 4.3. The point set of a symmetric plane has dimension $2^{n}$, where $1 \leqq n \leqq 4$. The pencil of all lines through a given point is a sphere of dimension $2^{n-1}$.

Proof. This has been proved for any stable plane whose lines are manifolds of positive dimension in [15: $\S 1]$. 
DEFINITION 4.4. For a symmetric plane $(M, \mathscr{L})$ with base point $o \in M$, let $T_{o} \mathscr{L}_{0}$ be the set of tangent spaces $T_{\text {, }} L \leqq T_{0} M$, where $L$ ranges over $\mathscr{L}_{0}$. Further denote by $T_{0} \mathscr{L}$ the union of all translates of $T_{o} \mathscr{L}_{o}$ in $T_{v} M$. Then the tangent plane $T_{o}(M, \mathscr{L})$ is defined to be the geometric structure

$$
T_{\diamond}(M, \mathscr{L})=\left(T_{0} M, T_{o} \mathscr{L}\right)
$$

together with the structure of Lie triple system present on $T_{v} M$.

Notation 4.5. For $\sigma \in \Sigma_{0}$, denote by $T_{v} \sigma$ the tangent map of the action of $\sigma$ on $M$, and by Ad $\sigma$ the action of $\sigma$ on $\subseteq$ given by $T_{1}\left(\gamma \rightarrow \sigma^{-1} \gamma \sigma\right)$. For $x \in \mathfrak{S}^{+}$, define ad $x: \subseteq \rightarrow \Im: y \rightarrow[y, x]$, and denote by ad $x \mid \mathfrak{S}^{-}$the restriction to $\mathfrak{S}^{-}$(cf. 3.3).

4.6. TheOREM B. (a) The tangent plane $T_{0}(M, \mathscr{L})$ of a symmetric plane $(M, \mathscr{L})$ is a topological translation plane, henceforth called the tangent translation plane.

(b) The line pencil $T_{u} \mathscr{L}_{0}$ through the origin consists of Lie triple subsystems of $T_{0} M$.

(c) For $\sigma \in \Sigma_{0}$, the mappings Ad $\sigma$ and $T_{0} \sigma$ coincide on $T_{0} M=\mathfrak{S}^{-}$. In particular, Ad $\sigma$ leaves $\mathcal{S}^{-}$invariant.

(d) The linear transformations Ad $\sigma \mid S^{-}=T_{0} \sigma$ of $T_{0} M$ (for $\left.\sigma \in \Sigma_{0}\right)$ are automorphisms of the translation plane $\left(T_{0} M, T_{0} \mathscr{L}\right)$ and of the Lie triple system.

(e) The identity component of the group $T_{v} \Sigma_{o}=\operatorname{Ad} \Sigma_{o} \mid \mathbb{S}^{-}$of automorphisms depends only upon the triple system $T_{0} M$. More precisely, its Lie algebra is ad $\mathfrak{S}^{+} \mid \mathfrak{S}^{-}$.

(f) The action of $\Sigma_{0}$ on $\mathfrak{S}^{-}$is faithful.

Proof. (1) If we are able to show that each nonzero $x \in T_{o} M$ lies on a unique tangent space $T_{0} L \in T_{0} \mathscr{L}_{0}$ then, from the fact that $\operatorname{dim} T_{0} M=2 \operatorname{dim} T_{0} L$, it follows easily that $T_{o}(M, \mathscr{L})$ is a (not necessarily topological) translation plane, cf. [11: VII. 3]. Obviously it suffices to prove the above assertion when $x$ belongs to some suitable neighborhood $W_{1} \subseteq T_{0} M$ of 0 .

(2) Let $W \subseteq T_{0} M$ be any convex open neighborhood of 0 on which Exp: $W \rightarrow \operatorname{Exp} W=U$ is a diffeomorphism (3.10). For $L \in \mathscr{L}_{\text {o }}$, Exp induces on $T_{o} L$ the exponential function of the subspace $L$, see 3.10a. Therefore the convex set $W \cap T_{0} L$, which is open in $T_{0} L$ and closed in $W$, is mapped under Exp onto a connected, closed and open subset $U_{L}$ of $L \cap U$. Then the union $V=U\left\{U_{L} ; L \in \mathscr{L}_{0}\right\}$ is an open neighborhood of $o$ by 4.1 , and $W_{1}=\operatorname{Exp}^{-1}(V)=U\left\{W \cap T_{0} L\right.$; $\left.L \in \mathscr{L}_{\text {o }}\right\}$ has the properties wanted in (1). 
(3) In order to show that the translation plane $T_{o}(M, \mathscr{L})$ is topological, we consider the map

$$
p: T_{0} M \backslash\{0\} \longrightarrow T_{0} \mathscr{L}_{0}: x \longrightarrow x \cup 0 .
$$

We endow $T_{0} \mathscr{L}_{0}$ with a Hausdorff topology by insisting that $\alpha: \mathscr{L}_{0} \rightarrow T_{0} \mathscr{L}_{0}: L \rightarrow T_{0} L$ be a homeomorphism. We show that $p$ is continuous and open with respect to this topology. It suffices to check this assertion on an exponential neighborhood, where it is equivalent to continuity and openness of $p^{\prime}: M \backslash\{0\} \rightarrow \mathscr{L}_{0}: x \rightarrow x \cup o$, since $\operatorname{Exp}$ and $\alpha$ are homeomorphisms. Finally, to see that $p^{\prime}$ is open, let $V$ be a neighborhood of $x \in M \backslash\{0\}$ such that $o \notin V$. Choose a line $K \in \mathscr{L}_{x} \mid \mathscr{L}_{0}$. By the stability axiom, $(V \cap K)^{p^{\prime}}$ is a neighborhood of $x \cup o$.

Since $p$ is continuous and open, $p$ is a quotient map. This implies that $T_{o}(M, \mathscr{L})$ is a topological translation plane [5: p. 34], since $T_{0} \mathscr{L}_{0}$ is Hausdorff by definition. Thus (a) is proved.

(4) Assertion (b) follows from 3.14.

(5) For $\sigma \in \Sigma_{0}$, the exponential map exp: $\subseteq \rightarrow \Sigma$ commutes with the automorphism $\theta=\theta_{\sigma}=\left(\gamma \rightarrow \sigma^{-1} \gamma \sigma\right)$ of $\Sigma$; that is $\operatorname{Ad} \sigma \circ \exp =$ $\exp \circ \theta$. This formula, together with those from 3.10, is used in the following computation, which proves (c) locally and hence globally:

$$
\operatorname{Exp}\left(x^{T_{o} \sigma}\right)=(\operatorname{Exp} x)^{\sigma}=o^{\sigma^{-1}(\exp x) \sigma}=o^{\exp \left(x^{\operatorname{Ad} \sigma}\right)}=\operatorname{Exp}\left(x^{\operatorname{Ad} \sigma}\right) .
$$

(6) It is clear that $T_{o} \sigma$ leaves $T_{o} \mathscr{L}_{0}$ invariant, for $\sigma \in \Sigma_{0}$. The rest of assertion (d) follows from 3.9.

(7) ad $\varsigma^{+}$is the Lie algebra of $\operatorname{Ad} \Sigma_{0}^{1}$. Therefore, Ad $\Sigma_{0}^{1}$ is generated by the elements exp ad $x$, where $x \in \mathfrak{S}^{+}$. Since both ad $x$ and $\exp$ ad $x$ leave the decomposition $\mathfrak{S}=\mathfrak{S}^{+} \oplus \mathfrak{S}^{-}$invariant, $\exp \left(\operatorname{ad} \mathfrak{S}^{+} \mid \mathfrak{S}^{-}\right)$generates $\operatorname{Ad} \Sigma_{o}^{1} \mid \mathfrak{S}^{-}$, whence (e). Note that $\mathfrak{S}^{+}$and ad $\mathfrak{S}^{+}$are determined by the triple system (3.8).

(8) Let $\sigma \in \Sigma_{0}$. Because $\sigma$ commutes with the exponential map (3.10), $T_{o} \sigma=$ id implies that $\sigma$ induces the identity on some open set, hence that $\sigma=1$.

The first step in the determination of all 4-dimensional symmetric planes will be to determine the possible 4-dimensional triple systems $T_{o} M$ (equivalently, the possible symmetric algebras (S, Ad $\left.\sigma_{o}\right)$ ), making use of the additional structure present on $T_{0} M$. Given $T_{0} M$, we shall then use the Ad $\Sigma_{0}$-invariance of $T_{0} \mathscr{L}_{0}$ in order to determine this set of sub-Lie triple systems. The final step will be to apply our Theorem C, which we approach next. Roughly speaking, Theorem $\mathrm{C}$ asserts that $(M, \mathscr{L})$ is uniquely determined by its linearized local structure $T_{o}(M, \mathscr{P})$. This is perhaps surprising if compared 
to the situation with symmetric spaces in general. It is in accordance, however, with other occurrences in geometry, cf. [7: §31]. The basic fact is that there are no proper covering morphisms of stable planes:

Definition 4.7. Let $(M, \mathscr{K})$ and $(N, \mathscr{L})$ be stable planes. A continuous map $\varphi: M \rightarrow N$ will be called a weak homomorphism of the planes, if for each connected component $A$ of a line $K \in \mathscr{K}$, the image $A^{\varphi}$ is contained in some line $L \in \mathscr{L}$. If each $K^{\varphi}$ is entirely contained in some line then we call $\varphi$ a homomorphism.

Proposition 4.8. Let $\varphi:(M, \mathscr{K}) \rightarrow(N, \mathscr{L})$ be a weak homomorphism of locally compact stable planes of finite positive dimension, which is a covering map of the point sets. Then each of the following conditions ensures that $\varphi$ is an isomorphism:

(a) Each line $L \in \mathscr{L}$ is connected and there exists a compact $D \in \mathscr{L}$.

(b) $\varphi$ is a homomorphism.

Before proving this result, we need the following lemma. Here, a space is said to have the domain invariance property if for each pair of homeomorphic subsets, either both of them, or neither of them are open, and a Cantor manifold is a separable metric space of dimension $n$ which is separated by no closed subset of a dimension $k<n-1$.

LEMMA 4.9. If a locally compact stable plane of finite positive dimension possesses a compact line then each line has the domain invariance property, and the compact lines are Cantor manifolds.

Proof. We shall show that each compact line is a homogeneous topological space. Since the lines are locally contractible metric [15: 1.12], they are ANR spaces [8]. Thus by a result of Eysko [19], the asserted properties follow for compact lines. Domain invariance carries over to all lines, since they may be projected homeomorphically onto open subsets of a compact line [15: 1.15]. (The quoted result says that a compact line meets every other line.)

Now let $L$ be a compact line, and $x, y \in L$. By [15: 1.16], there is a compact line $K$ meeting $L$ in a point $w \neq x, y$. Again, using [15: 1.15], it is easy to project $L$ onto $K$ and back onto itself in such a fashion that $x$ goes to $y$.

Proof of 4.8. (1) First assume condition (b). If there exist $x_{1} \neq x_{2} \in M$ with $x_{1}^{\varphi}=x_{2}^{\varphi}$, choose points $z, w$, such that $z \notin x_{1} \cup x_{2}$, 
$z^{\varphi} \neq x_{i}^{\varphi}$, and $w^{\varphi} \notin L$, where $L$ is the line containing the images of $K_{i}=x_{i} \cup z$. For $y_{1} \in K_{1}$ near $z$, we have $y_{1}^{\varphi}=y_{2}^{\kappa}$, where $y_{2}:=$ $\left(w \cup y_{1}\right) \cap K_{2}$ is the projection from $w$. This is impossible, since $\varphi$ is injective near $z$. Thus $\varphi$ must be a homeomorphism. By similar arguments, $\varphi$ is easily seen to be injective on each pencil $\mathscr{K}_{x}$ and, hence, on $\mathscr{C}$. This means that $\varphi$ is a collineation in the sense of [15], hence an isomorphism [15: p. 256].

(2) Next assume condition (a). We begin by showing that every line $L \in \mathscr{L}$ contains the image $A^{\varphi}$ of some component of some line $K \in \mathscr{K}$, and that moreover $\varphi$ induces homeomorphisms between the line pencils $\mathscr{K}_{x}$ and $\mathscr{L}_{x} \varphi$. To do this, consider a neighborhood $U$ of $x$ such that $\varphi \mid U$ is an open embedding, and construct from $U$ a neighborhood $V$ as in 4.1. $\mathscr{K}_{x}$ and $\mathscr{L}_{x}$ are the quotient spaces of the pierced neighborhoods $V \backslash\{x\}$ and $V^{\varphi} \backslash\left\{x^{\varphi}\right\}$, with respect to the continuous and open mappings $p: y \rightarrow y \cup x$ and $q: z \rightarrow z \cup x^{\varphi}$. Now by the choice of $V, \varphi$ commutes with $p$ and $q$, and by the argument employed in (1), the factorization $\varphi^{\prime}: \mathscr{K}_{x} \rightarrow \mathscr{L}_{x}^{\varphi}$ is injective; the above assertions follow. Note that this implies that any two lines $K \in \mathscr{K}$ and $L \in \mathscr{P}$ are locally homeomorphic and thus extends the applicability of domain invariance (4.9).

(3) If $A$ is a component of $K \in \mathscr{K}$, then $A^{\varphi} \in \mathscr{L}$. Indeed, we know that $A^{\varphi}$ is contained in a line $L \in \mathscr{L}$; it suffices to show that the closure $\overline{A^{\varphi}}$ is contained in the interior of $A^{\varphi}$ relative to $L$. For $x \in \overline{A^{\varphi}}$, choose an open neighborhood $U$ of $x$ in $N$ which has the covering property, and for which $W=U \cap L$ is connected. Let $T$ be a sheet of $U^{\varphi^{-1}}$ such that $T \cap A \neq \varnothing$. Since $\varphi$ maps $T$ homeomorphically onto $U$, and by domain invariance, $(T \cap A)^{\varphi}$ is a closed and open subset of $W$. Hence, $W \cong A^{\varphi}$.

Note that, moreover, $A$ is mapped homeomorphically onto $A^{\varphi}$. Indeed, if $x_{1}^{\varphi}=x_{2}^{\circ}$ for different points $x_{i} \in A$ then for $z$ sufficiently close to $A$, the points $x_{i}$ and $z$ are in the same component of $x_{i} \cup z$. (Use a path in $A$ joining $x_{1}$ to $x_{2}$ and project it into $x_{i} \cup z$.) This enables us to obtain a contradiction by the argument of (1).

(4) Applying (3) to $D$, the given compact line, we obtain a line $K \in \mathscr{Y}$ and a component $A$ of $K$ such that $A \cong D$. Then $A=K$ because, embedding $K$ into $\mathscr{L}_{p}$ for $p \notin K$ via $\cup p=(x \rightarrow x \cup p)$, one gets $A^{\cup p} \cong K^{\cup p} \cong \mathscr{L}_{p}$, and $A^{\cup p}=\mathscr{L}_{p}$ by domain invariance and by connectedness of $\mathscr{L}_{p}[15: 1.14]$. Thus we have proved that the set $\mathscr{C} \subseteq \mathscr{K}$ of compact lines is nonempty. It is further open by [15: 1.16].

(5) We are now ready to prove that $\phi$ is one-to-one, hence a homeomorphism. It suffices to show that a point $x_{1}$ on a compact (hence connected) line $C_{1} \in \mathscr{K}$ satisfies $x_{1}^{\varphi \varphi-1}=\left\{x_{1}\right\}$. Assume to the contrary that $x_{1}^{\circ}=x_{2}^{\varphi}$ for $x_{2} \neq x_{1}$. By (2) and (3), the line $C_{1}^{\varphi}$ is also 
the (homeomorphic) image of some line $C_{2} \in \mathscr{K}_{x_{2}}$, necessarily different from $C_{1}$. By [15: 1.15], the lines $C_{1}$ and $C_{2}$ meet in a point $z$, and $z^{\varphi} \neq x_{i}^{\varphi}$. Again, a contradiction follows as in (1).

(6) Combining (2), (3) and (5), we see that we may identify $M$ and $N$ and that $\mathscr{L}$ consists of the connected components of the elements of $\mathscr{K}$. Assume finally that some $K \in \mathscr{K}$ is disconnected. Two points $x$ and $y$ in different components of $K$ may then be joined by some line $L \in \mathscr{L}$, and there is a $K^{\prime} \in \mathscr{\mathscr { C }}$ such that $L \cong K^{\prime}$. Then necessarily $K \neq K^{\prime}$, a contradiction.

REMARK 4.10. If in $4.8 \mathrm{a}$ we drop the requirement that all lines of $N$ be connected, then the same proof goes through to show that $\varphi$ is one-to-one and that the set of all connected components of $\mathscr{K}$ lines and the set of components of $\mathscr{L}$-lines are the same. Thus the $\mathscr{P}$-lines are obtained from the $\mathscr{K}$-lines by reshuffling their connected components. It is not, in general, possible to deduce more than this. For an illustration, consider the following.

ExAmple. Let $M=\{(x, y) ; y \neq 0$ or $x<0\} \cong \boldsymbol{R}^{2}$. In order to obtain a new line system $\mathscr{C}$ for this plane, recombine the components of the ordinary lines $L \in \mathscr{L}$ as follows: Form a new line by pairing, for each $x \geqq 0$, the upper part of each line through the point $(x, 0)$ with the lower part of its parallel through $(2 x, 0)$. Then $\varphi:=$ id: $(M, \mathscr{K}) \rightarrow(M, \mathscr{L})$ is a weak homomorphism, but not a homomorphism.

4.11. THeOREM C. Let $(M, \mathscr{K})$ and $(N, \mathscr{L})$ be connected symmetric planes and let $\psi: T_{0}(M, \mathscr{K}) \rightarrow T_{0}(N, \mathscr{L})$ be an isomorphism (of the triple systems and the translation planes). Assume that (a), (b) or (c) holds:

(a) $M$ is simply connected and all lines of $M$ are connected.

(b) $N$ has trivial centre, and all lines of $M$ are connected.

(c) $N$ has trivial centre, has connected lines only and contains at least one compact line.

Then $(M, \mathscr{K})$ and $(N, \mathscr{L})$ are isomorphic symmetric planes.

Proof. By 3.12, there is a covering morphism of symmetric spaces $\varphi: M \rightarrow N$ such that $T_{\circ} \varphi=\psi$. Theorem $\mathrm{C}$ follows from 4.8 together with the following lemma:

Lemma 4.12. Let $(M, \mathscr{K})$ and $(N, \mathscr{L})$ be connected symmetric planes and $\varphi: M \rightarrow N$ a morphism of the underlying symmetric spaces. If $T_{\circ} \varphi$ is an isomorphism of the tangent translation planes 
then $\varphi$ is a weak homomorphism of stable planes.

Proof. By hypothesis, $\varphi$ is a covering morphism of the spaces.

(1) For $K \in \mathscr{K}_{0}$, let $A$ be the connected component of $o$ in $K$. Then $A$ is a symmetric subspace of $M$ with tangent space $T_{0} K$. By our assumption, $T_{o} \varphi$ maps $T_{o} K$ to some $T_{o} L \in T_{o} \mathscr{L}_{0}$, and $\varphi$ maps $A$ to a symmetric subspace of $N$ with the same tangent space as $L$. By $3.14, A^{\varphi} \subseteq L$.

(2) Let $\sigma \in \Sigma_{M}$ be a motion of $M$. Then there is a motion $\delta$ of $N$ such that $\sigma \varphi=\varphi \delta$. This is easily seen in the case of a symmetry $\sigma=\sigma_{y}$ : then $\sigma_{y} \varphi=\varphi \sigma_{w}$, where $w=y^{\varphi}$.

(3) Now let $B$ be any component of any line $G \in \mathscr{K}$. For an arbitrary $b \in B$, choose a motion $\sigma \in \Sigma_{M}$ with $o^{\sigma}=b$. Then $B=A^{\sigma}$, where $A$ is a component has in (1). Then by (2), $B^{\varphi}=A^{\sigma \varphi}=A^{\varphi \delta}$ is contained in a line.

REMARK 4.13. By 4.10, the condition that the lines of $N$ be connected can be dropped in 4.11c, the following weaker assertion remaining valid: There exists a weak homomorphism $\varphi: M \rightarrow N$ of the stable planes which is an isomorphism of symmetric spaces and induces a bijection of the sets of connected components of lines.

4.14. THEOREM D. For a connected symmetric plane $(M, \mathscr{L})$, the following are equivalent:

(a) $(M, \mathscr{L})$ is an affine plane.

(b) $(M, \mathscr{L})$ is an affine translation plane.

(c) $T_{0} M$ is an abelian triple system, that is, has trivial multiplication.

(d) $\Sigma^{+}$is abelian.

(e) $\Sigma_{0}$ is discrete.

(f) $M$ is an abelian symmetric space; that is, the centre congruence is trivial: $C(M)=M \times M$.

Proof. By a result of R. Baer [21: p. 213], (b) follows from (a). Alternatively, one could use 3.1 to obtain transitivity of the group of translations.

Clearly, (b) implies (c). For the converse implication, note that for any symmetric plane, the plane $T_{o} T_{o}(M, \mathscr{L})$ is isomorphic to $T_{o}(M, \mathscr{L})$ as a translation plane, but has trivial triple structure. Therefore, if (c) holds,

$$
T_{o} T_{o}(M, \mathscr{L}) \cong T_{o}(M, \mathscr{L}),
$$

which implies $T_{0}(M, \mathscr{L}) \cong(M, \mathscr{L})$ by 4.11a. 
Let $\left(\mathrm{e}^{\prime}\right)$ be the property $\mathfrak{S}=\mathfrak{S}^{-}$, which is clearly equivalent to condition (e). We get equivalence of (c), (d), and ( $\left.e^{\prime}\right)$ from the facts that $\left[\mathfrak{S}^{-}, \mathfrak{S}^{-}\right]=\mathfrak{S}^{+}(3.5 \mathrm{a})$ and that $\mathfrak{S}^{\text {is }}$ the standard embedding of $T_{o} M$ 3.8. Finally, (d) and (f) equivalent by (3.11).

4.15. THEOREM E. Each of the symmetric planes described as Examples 2.5 through 2.13, with the possible exception of the complex Minkowski plane, is isomorphic to any other connected symmetric plane which has the same tangent translation plane (comprising the triple structure).

REMARK. The same assertion does hold for the Minkowski plane, as will be shown in [17], using condition (b) of Theorem C.

Proof. Each of the examples has connected lines. A symmetric plane has trivial centre if its motion group $\Sigma^{+}$is centre-free, since $\sigma_{x}=\sigma_{y}$ implies that $\sigma_{x}$ is a reflection at both $x$ and $y$, hence that $x=y$. Thus the real and complex elliptic, exterior hyperbolic and punctured projective planes satisfy the criterion (c) of Theorem C. All affine planes, as well as the real and complex interior hyperbolic and cylinder planes, are simply connected and satisfy the first criterion of Theorem $C$. The only remaining example, the complex oval plane $M$, needs special treatment.

$M$ is not simply connected, as will be seen in the course of our argument, and is centre-free, because $\Sigma=\mathrm{PSO}_{3} C=\Sigma^{+}$contains the reflections, hence has no centre. It has no compact line, since each complex line meets the orthogonal quadric $P_{2} C \backslash M$. Thus $M$ satisfies none of our criteria.

To show that, nevertheless, $M$ satisfies our assertion, we shall prove that the universal covering $\widetilde{M} \rightarrow M$ is two-sheeted, and that $\widetilde{M}$ has the same motion group $A \cong \Sigma$, with a smaller isotropy group $\Lambda_{0}<\Sigma_{0}$. Thus $\Lambda_{0}$ must be different from the centralizer $\Sigma_{0}$ of $\sigma_{o}$, and $\tilde{M}$ cannot be a symmetric plane by 1.6. Therefore, for any symmetric plane $M^{\prime}$ with the same tangent translation plane as $M$, there is a bijective weak homomorphism $\varphi: M \rightarrow M^{\prime}$. Now $M$ has connected lines, and $\varphi$ is an isomorphism by $4.8 \mathrm{~b}$.

By computation one finds $\Sigma_{o} \cong \mathrm{O}_{2} C \cong \Omega \cdot\langle\alpha\rangle$, where $\Omega$ is the multiplicative group of complex numbers and $\alpha$ is the automorphism $z \rightarrow z^{-1}$ of $\Omega$. The symmetric space $N:=\Sigma / \Omega$ is a two-sheeted connected covering of $M=(\Sigma / \Omega) /\left(\Sigma_{o} / \Omega\right)$. Our proof is complete as soon as we show that $N$ is simply connected (hence that $N=\tilde{M}$ ).

By exactness of the homotopy sequence of the fibration $\Sigma \rightarrow N$, it suffices to show that $\pi_{1} \Omega \rightarrow \pi_{1} \Sigma$ is surjective. To verify this, let $T \leqq \Gamma$ be maximal compact subgroups of $\Omega$ and $\Sigma$, respectively. 
Then $\Gamma \cong \mathrm{SO}_{3} \boldsymbol{R}$, and by the theorem of Malcev-Iwasawa [20: p. 189], we only have to show that $\pi_{1} T \rightarrow \pi_{1} \Gamma$ is surjective. This may be read off from the homotopy sequence of the fibration associated with the transitive action of $\Gamma$ on the 2 -sphere.

\section{Determination of all 2-dimensional symmetric planes.}

5.1. Determination of all reflection algebras of type (p, 2). Reflection algebras are defined in 3.7. For our present purposes it would suffice to have a list of the effective ones. However in [17], it will prove useful to know slightly more than that. Since a reflection algebra satisfies the dimension estimate $3.5 \mathrm{~b}$, we must have $p \leqq 1$ for each reflection algebra $(\Re, \alpha)$ of type $(p, 2)$. We find

(1) One abelian reflection algebra (,$\alpha)$ of type $(p, 2)$, characterized by $p=0$.

If $p=1$, then $\Re$ is simple or solvable [12: p. $11 \mathrm{ff}]$. The subspace $\Re^{+}$is orthogonal to $\Re^{-}$with respect to the Killing form of $\Re$, see [14: p. 140]. Therefore, if $\Re$ is simple, $\alpha$ is determined by the one-dimensional subspace $\Re^{+}<\Re$. Now let $\Re=\subseteq \mathfrak{\Omega}_{3}$ be the Lie algebra of $\Sigma=\mathrm{SO}_{3} R$. Then the adjoint representation of $\Sigma$ is the standard action on $\boldsymbol{R}^{3}$ and is transitive on one-dimensional subspaces of $\Re$. Hence there is at most one such algebra $\left(\subseteq \Omega_{3}, \alpha\right)$. One the other hand, the algebra of the real elliptic plane (2.13) is of this kind. So we have

(2) One reflection algebra $\left(5 \mathfrak{D}_{3}, \alpha\right)$ of type $(1,2)$.

If $\Re=\mathfrak{S R}_{2} R$ is the Lie algebra of $\mathrm{SL}_{2} R$, then the adjoint group is the identity component of $\mathrm{SO}_{3}(\boldsymbol{R}, 1)=\mathrm{SO}_{3}(f)$, where $f$ is the Killing form of $\Re$, and has three orbits of one-dimensional subspaces. Among these, the one-dimensional orbit, consisting of the selforthogonal subspaces, gives no reflection algebras, since $\mathfrak{R}^{+}$and $\Re^{-}$have to be complementary. Thus, in view of the two examples associated with the real hyperbolic planes (2.13), we have

(3) Two nonisomorphic reflection algebras $\left(\subseteq \mathbb{R}_{2}, \alpha\right)$ of type $(1,2)$. The subgroup $\Sigma_{o} \leqq \mathrm{PSL}_{2} R$ corresponding to $\Re^{+}$is compact in one case and noncompact in the other.

It remains to consider the solvable reflection algebras of type $(1,2)$. Let $\Omega \leqq \Re$ be the commutator subalgebra. Then by (3.5a), $\Re$ contains $\Re^{+}$. Furthermore, we have $1 \leqq \operatorname{dim} \Omega \leqq 2$, and $\Re$ is abelian [12: p. 12]. If $\Omega$ is one-dimensional, then $\Omega=\Re^{+}$must be central because $\left[\Re^{+}, \Re^{-}\right] \subseteq \Re^{-}$. In this case, a basis $\{a, b, c\}$ of $\Re$ may be found such that

$$
\mathfrak{R}^{+}=\langle a\rangle, \mathfrak{R}^{-}=\langle b, c\rangle, \quad a=[b, c] .
$$


Thus we have

(4) One nilpotent reflection algebra $(\Re, \alpha)$ of type $(1,2) ; \Re^{+}$ is the centre of $\Re$, and $(\Re, \alpha)$ is not effective in the sense of 3.7.

If $\operatorname{dim} \Re=2$, then $\Re=\Omega^{+} \oplus \Re^{-}=\langle a\rangle \oplus\langle b\rangle$, say, since $\Re$ is $\alpha$-invariant. Choose $c \in \Re^{-} \mid \Re^{-}$. Then ad $c$ interchanges $\Re^{+}$and $\Re^{-}$, and is, by the definition of $\Re$, an automorphism of $\Re$. Thus ad $c$ may be represented by a regular matrix of the form

$$
\left(\begin{array}{ll} 
& r \\
s &
\end{array}\right)
$$

with respect to the basis $\{a, b\}$ of $\AA$. Replacing $c$ and $a$ by suitable scalar multiples, one may reduce the pair $(r, s)$ to $(1,1)$ or $(1,-1)$. The case $s=-1$ gives

(5) One reflection algebra $(\Re, \alpha)$ of type $(1,2)$, where $\Re$ is the Lie algebra of the euclidean motion group $\boldsymbol{R}^{2} \cdot \mathrm{SO}_{2}$, and $\Re^{+}$is contained in the commutator subalgebra.

Finally, for $s=1$, ad $c$ is equivalent to the endomorphism $(x, y) \rightarrow(x,-y)$ of $\Re$, and we have

(6) One reflection algebra $(\Re, \alpha)$ of type $(1,2)$, where $\mathfrak{R}$ is the Lie algebra of the motion group of the real cylinder plane, see 2.13 , and $\mathfrak{R}^{+}$is contained in the commutator subalgebra.

5.2. THEOREM F. The list 2.13 of 2-dimensional connected symmetric planes is complete.

Proof. In a 2-dimensional affine topological translation plane, the set of lines through the origin is necessarily the set of all onedimensional real subspaces. This, together with Theorem E, shows that each of the planes listed in 2.13 is uniquely determined by its Lie triple system. Since by 3.8 the classification of $n$-dimensional triple systems is equivalent to the classification of all effective reflection algebras of type $(p, n)$ with arbitrary $p$, the proof of Theorem $\mathrm{F}$ boils down to showing that for $n=2$, this latter classification yields exactly the reflection algebras ( $\subseteq, \operatorname{Ad} \sigma_{o}$ ) associated with the planes 2.13. This is easily verified by comparing 2.13 with 5.1.

\section{REFERENCES}

1. R.F. Arens, A topology for spaces of homeomorphisms, Ann. Math., 47 (1946), 480-495.

2. D. Betten, 4-dimensionale Translationsebenen, Math. Z., 128 (1972), 129-151.

3. - 4-dimensionale Translationsebenen mit kommutativer Standgruppe, Math.

Z., 154 (1977), 125-141.

4. - Die komplex-hyperbolische Ebene, Math. Z., 132 (1973), 249-259. 
5. P. Breuning, Translationsebenen und Vektorraumbündel, Mitt. Math. Sem. Gießen, 86 (1970).

6. T. Buchanan, Ovale und Kegelschnitte in der komplexen projektiven Ebene, Math. Phys. Sem. Ber., 26 (1979), 244-260.

7. H. Busemann, The Geometry of Geodesics, New York: Academic Press, 1955.

8. J. Dugundji, Absolute neighborhood retracts and local connectedness in arbitrary metric spaces, Comp. Math., 13 (1958), 229-246.

9. H. Hähl, Automorphismengruppen achtdimensionaler lokalkompakter Quasikörper, Math. Z., 149 (1976), 203-225.

10. G. Hochschild, The Structure of Lie Groups, San Francisco: Holden Day, 1965.

11. D. R. Hughes and F.C. Piper, Projective Planes, New York, Heidelberg, Berlin: Springer, 1973.

12. N. Jacobson, Lie Algebras, New York-London: Interscience, 1962.

13. E. N. Kuzmin, On some classes of division algebras, Algebra i Logika 5 (2) (1966), 57-102 (Russian).

14. O. Loos, Symmetric Spaces, I, New York: Benjamin, 1969.

15. R. Löwen, Vierdimensionale stabile Ebenen, Geom. Dedic., 5 (1976), 239-294.

16. - Halbeinfache Automorphismengruppen von vierdimensionalen stabilen Ebenen sind quasi-einfach, Math. Ann., 236 (1978), 15-28.

17. Classification of 4-dimensional symmetric planes, Math. Z., 167 (1979), 137-159.

18. - Characterization of symmetric planes of dimension at most 4, to appear. 19. J. M. Lysko, Some theorems concerning finite-dimensional ANR-spaces, Bull. Acad. Pol. Sci., Sér. Sci. Math. Astron. Phys., 24 (1976), 491-496.

20. D. Montgomery and L. Zippin, Topological Transformation Groups, New York: Interscience, 1955.

21. G. Pickert, Projektive Ebenen, 2nd ed., Berlin, Heidelberg, New York: Springer, 1975.

22. H. Salzmann, Topological planes, Advances in Math., 2 (1967), 1-60.

23. P. A. Smith, Fixed point theorems for periodic transformations, Amer. J. Math., 63 (1941), 1-8.

24. K. Strambach, Salzmann-Ebenen mit hinreichend vielen Punkt oder Geradenspiegelungen, Math. Z., 99 (1967), 247-269.

25. - Zentrale und axiale Kollineationen in Salzmann-Ebenen, Math. Ann., 185 (1970), 173-190.

26. J. Szenthe, On the topological characterization of transitive Lie group actions, Acta Sci. Math. Szeged., 36 (1974), 323-344.

27. J. Tits, Tabellen zu den einfachen Liegruppen und ihren Darstellungen, Lecture Notes in Math., 40 Berlin, Heidelberg, New York: Springer, 1969.

Received November 29, 1978.

Mathematisches INSTITUT DER UNIVERSität Auf Der Morgenstelle 10, D-74 TÜBIngen BundesRepublic Deutschland 


\section{PACIFIC JOURNAL OF MATHEMATICS}

\section{EDITORS}

DONALD BABBITT (Managing Editor)

University of California

Los Angeles, CA 90024

HUGO RossI

University of Utah

Salt Lake City, UT 84112

C. C. Moore and ANDrew OGG

University of California

Berkeley, CA 94720
J. DUGUNDJI

Department of Mathematics University of Southern California Los Angeles, CA 90007

R. FINN and J. MILGRAM

Stanford University

Stanford, CA 94305

\section{ASSOCIATE EDITORS}
E. F. BeCKENBACH
B. H. NeumanN
F. WOLF
K. YOSHIDA

\section{SUPPORTING INSTITUTIONS}

UNIVERSITY OF BRITISH COLUMBIA CALIFORNIA INSTITUTE OF TECHNOLOGY UNIVERSITY OF CALIFORNIA MONTANA STATE UNIVERSITY UNIVERSITY OF NEVADA, RENO NEW MEXICO STATE UNIVERSITY OREGON STATE UNIVERSITY UNIVERSITY OF OREGON

\author{
UNIVERSITY OF SOUTHERN CALIFORNIA \\ STANFORD UNIVERSITY \\ UNIVERSITY OF HAWAII \\ UNIVERSITY OF TOKYO \\ UNIVERSITY OF UTAH \\ WASHINGTON STATE UNIVERSITY \\ UNIVERSITY OF · WASHINGTON
}

The Supporting Institutions listed above contribute to the cost of publication of this Journal, but they are not owners or publishers and have no responsibility for its content or policies.

Mathematical papers intended for publication in the Pacific Journal of Mathematics should be in typed form or offset-reproduced, (not dittoed), double spaced with large margins. Please do not use built up fractions in the text of the manuscript. However, you may use them in the displayed equations. Underline Greek letters in red, German in green, and script in blue. The first paragraph or two must be capable of being used separately as a synopsis of the entire paper. Please propose a heading for the odd numbered pages of less than 35 characters. Manuscripts, in triplicate, may be sent to any one of the editors. Please classify according to the scheme of Math. Reviews, Index to Vol. 39. Supply name and address of author to whom proofs should be sent. All other communications should be addressed to the managing editor, or Elaine Barth, University of California, Los Angeles, California, 90024.

50 reprints to each author are provided free for each article, only if page charges have been substantially paid. Additional copies may be obtained at cost in multiples of 50 .

The Pacific Journal of Mathematics is issued monthly as of January 1966. Regular subscription rate: $\$ 84.00$ a year (6 Vols., 12 issues). Special rate: $\$ 42.00$ a year to individual members of supporting institutions.

Subscriptions, orders for numbers issued in the last three calendar years, and changes of address should be sent to Pacific Journal of Mathematics, P.O. Box 969, Carmel Valley, CA 93924, U.S.A. Older back numbers obtainable from Kraus Periodicals Co., Route 100, Millwood, NY 10546.

PUBLISHED BY PACIFIC JOURNAL OF MATHEMATICS, A NON-PROFIT CORPORATION

Printed at Kokusai Bunken Insatsusha (International Academic Printing Co., Ltd.). 8-8, 3-chome, Takadanobaba, Shinjuku-ku, Tokyo 160, Japan.

Copyright (C) 1979 by Pacific Journal of Mathematics Manufactured and first issued in Japan 


\section{Pacific Journal of Mathematics}

\section{Vol. 84, No. $2 \quad$ June, 1979}

Somesh Chandra Bagchi and Alladi Sitaram, Spherical mean periodic

functions on semisimple Lie groups ........................ 241

Billy Joe Ball, Quasicompactifications and shape theory............. 251

Maureen A. Bardwell, The o-primitive components of a regular ordered permutation group ................................ 261

Peter W. Bates and James R. Ward, Periodic solutions of higher order

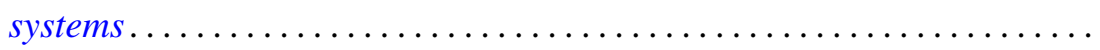

Jeroen Bruijning, A characterization of dimension of topological spaces by totally bounded pseudometrics......................... 283

Thomas Farmer, On the reduction of certain degenerate principal series

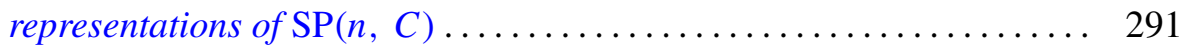

Richard P. Jerrard and Mark D. Meyerson, Homotopy with m-functions . . . . 305

James Edgar Keesling and Sibe Mardesic, A shape fibration with fibers of

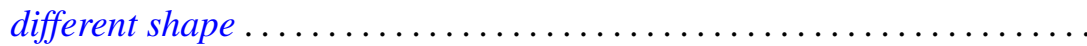

Guy Loupias, Cohomology over Banach crossed products. Application to bounded derivations and crossed homomorphisms ...............

Rainer Löwen, Symmetric planes ........................ 367

Alan L. T. Paterson, Amenable groups for which every topological left

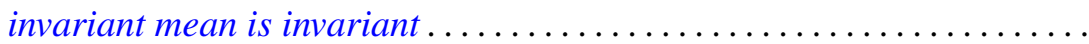

Jack Ray Porter and R. Grant Woods, Ultra-Hausdorff H-closed extensions

Calvin R. Putnam, Operators satisfying a $G_{1}$ condition .

Melvin Gordon Rothenberg and Jonathan David Sondow, Nonlinear smooth representations of compact Lie groups..............

Werner Rupp, Riesz-presentation of additive and $\sigma$-additive set-valued measures.

A. M. Russell, A commutative Banach algebra of functions of generalized variation

Judith D. Sally, Superregular sequences

Patrick Shanahan, On the signature of Grassmannians . . 